\title{
Relación entre el índice de masa corporal y la competencia percibida en adolescentes de la Educación Secundaria Obligatoria Relation between de body max index and the perceived motor competence in secondary education
}

\author{
Saioa Urrutia Gutierrez*, Susana Irazusta Adarraga*, Amaia Balerdi Iztueta**, Oscar González Rodríguez**, \\ JoséAntonio Arruza Gabilondo*** \\ *Universidad del País Vasco, ** Grupo iceberg S.L., ***TESKAL
}

\begin{abstract}
Resumen: En esta sociedad, caracterizada por el desarrollo del conocimiento, de las tecnologías de la comunicación y de la generalización de los medios de transporte, los niveles de actividad física en la vida cotidiana han descendido. Esto ha traído un aumento del sedentarismo, trastornos relacionados con la salud y la alimentación y obesidad. Diferentes investigaciones han mostrado que tales co-morbilidades pueden derivarse del descenso del nivel de competencia motriz que se viene produciendo en los últimos años y su relación con la percepción de la misma. Ante esta situación, nos hemos propuesto determinar la relación entre el índice de masa corporal y la percepción de competencia. 366 sujetos de 13 a 16 años han participado en estudio. Se ha medido el peso (kgs y la altura (metros) de los sujetos y se les ha aplicado el cuestionario AMPET-R. Los resultados muestran que no hay diferencias significativas en cuanto al índice de masa corporal y que los chicos obtienen valores más altos en Percepción de competencia motriz que las chicas. Por otro lado, se ha comprobado que el índice de masa corporal y la percepción de competencia se relacionan significativa y negativamente, tanto para los chicos como para las chicas.
\end{abstract}

Palabras clave: Competencia motriz percibida; Índice de Masa Corporal; Sobrepeso; Obesidad.

\begin{abstract}
This society, characterized by the development of knowledge, communication technologies and the generalization of transports, levels of physical activity in daily life have been declined. This has increased the sedentary lifestyle, health and nutrition related disorders and obesity. Different studies have demonstrated that this kind of co-morbidities may result from the decreased level of motor competence that has been occurring in recent years and the relation to the perception of it. At this point, we proposed to determine the relationship between body mass index and perceived motor competence. 366 subjects from 13 to 16 years have participated in this study. The weight $(\mathrm{kg})$ and height (meters) of participants was measured while they were answering the AMPET-R questionnaire. The results show that there are not significant differences between different levels of body mass index and boys obtained higher values for perceived motor competence than girls. On the other hand, it has been show that body mass index and perceived motor competence are related significantly but negatively, for both girls and boys.
\end{abstract}

Key words: Perceived motor competence; body mass index; overweight; obesity.

\section{Introducción}

El descenso de los niveles de actividad física en la vida cotidiana que se da en esta sociedad marcada por el desarrollo y generalización de las tecnologías de comunicación y los medios de transporte. Sin duda, la escasa importancia que el ejercicio o actividad física cotidiana tiene en nuestra sociedad, aumenta los problemas relacionados con el sedentarismo, sobrepeso y la obesidad, evolucionando en problemas de salud pública, clínicos y económicos (Lakka \& Bouchard, 2005).

Por lo tanto, la falta de necesidad de realizar una actividad física ligada a la vida cotidiana conlleva que la práctica para un bueno mantenimiento de la salud, se realice en el tiempo de ocio. La falta de tiempo de ocio y la alimentación no planificada y descontrolada de los jóvenes, trae como consecuencia un aumento del sobrepeso y del sedentarismo, con un descenso generalizado de los indicadores de salud (Santos, 2006; Solbes, 2010)

Uno de los indicadores más afianzados es el expuesto por Quetelet (1853): El índice de masa corporal (IMC) o también conocido como el índice de Quetelet. Es la medida de asociación entre el peso y la altura de una persona, que se obtiene dividiendo el peso (kilogramos) por la altura al cuadrado (metros).

En base a este índice, La Organización Mundial de la Salud, dependiendo del valor del IMC, estableció las siguientes categorías: Infrapeso, Normopeso, Sobrepeso y Obeso. A su vez, en el año 1997 definió que a partir de los 18 años con un IMC que oscila entre $18.5 \mathrm{Kg}$./ $\mathrm{m}^{2}$ y $24.9 \mathrm{Kg} . \mathrm{m}^{2}$ se considera Normopeso, el Sobrepeso a partir de 25 $\mathrm{Kg} . \mathrm{m}^{2}$ y la Obesidad a partir de $30 \mathrm{Kg} . / \mathrm{m}^{2}$.

Hoy en día, los valores de estas dos últimas categorías (Obesidad y Sobrepeso) están alcanzado unos valores alarmantes, especialmente en

Fecha recepción: 01-07-12- Fecha envío revisores: 01-07-12- Fecha de aceptación: 13-12-12 Correspondencia: Saioa Urrutia Gutierrez

Plaza Oñati, 3

20018 Donostia-San Sebastián (Gipuzkoa

Email: saioaurrutiagutierrez@gmail.com la niñez y en la adolescencia, dónde cada vez es más fácil padecer este trastorno. Tanto es así, que en el año 2004 la OMS ya declaró que la obesidad había alcanzado el carácter de pandemia (Eaton, et al., 2006; Solbes, 2010).

Debido a la alarmante situación de los niveles de Obesidad y Sobrepeso, durante muchos años se ha estado buscando medios para poder solventar y buscar la raíz de este problema. Se han desarrollado varias investigaciones (Bueno, Ruiz, Graupera \& Sánchez, 2001; Gómez, 2004; Ruiz \& Mata, 2008), que han mostrado que tales co-morbilidades como la obesidad y el sobrepeso, pueden derivarse del descenso del nivel de competencia motriz que se viene produciendo en los últimos años.

Por lo tanto, el alumnado con este tipo de problemas y que además no es competente muestra problemas en el aprendizaje y mejora de sus capacidades (Dunn \& Watkinson, 1994). Esto acarrear una evaluación de sus habilidades, lo que da lugar a crear una imagen del sujeto basada en la percepción de su competencia, es decir, la creación de la percepción de la competencia motriz (PCM). Arruza et al. (2011) definen la competencia motriz como la creencia que tiene cada persona de su nivel dehabilidad.

De esta manera, sehan desarrollado diferentes investigaciones(Dunn \& Watkinson, 1994; Jones, Okely, Caputi \& Cliff, 2010; Southall, Steele \& Okely, 2004), en las que se ha observado la estrecha relación entre la percepción y la competencia real. La influencia en la creación de la percepción de competencia motriz se basa en los éxitos y fracasos acumulados a la hora derealizar cualquier tipo de actividad físico-deportiva y por lo tanto una buena realización de actividades motoras conlleva una alta percepción de competencia motriz. En cambio, una mala ejecución acarrea una baja percepción de competencia motriz (Harter, 1978, 1981).

Los resultados de la investigación llevada a cabopor los investigadores Weiss y Amorose (2005) indican que percepción de competencia motriz es una variable importante para explicar los procesos de motivación hacia la práctica físico-deportiva, que a su vez influyen en los niveles de IMC. 
En este sentido, la finalidad de este proyecto es conocer y describir la posible relación entre el IMC y la Percepción de competencia motriz.

\section{Método}

\section{Participantes}

En este estudio participaron 366 estudiantes de cinco ikastolas diferentes, en la etapa educativa de Enseñanza Secundaria Obligatoria, pertenecientes al Territorio Histórico de Gipuzkoa de la Comunidad Autónoma Vasca. Los datos fueron obtenidos de alumnos y alumnas que oscilaban entre los 13 y los 16 años de edad.

\section{Instrumentos}

Para alcanzar este objetivo se emplearon los siguientes instrumentos:

a) Competencia Percibida: La competencia Percibida fue medida mediante el test AMPET-R de Ruiz, Graupera y Rico (2004), que mide lamotivación de logro. Dentro de la motivación de logro hay tres variables; la ansiedad frente al error, el compromiso y la competencia motriz percibida. Está última escala fue la que se uso para medir el nivel de competencia percibida.

b) Índice de Masa Corporal (IMC): Para la medición del IMC, se tomó el peso y la altura del alumnado y después se aplico la siguiente fórmula para poder conseguir el IMC (IMC=Peso/Altura2).

\section{Procedimiento}

Se realizo una previa selección de los centros y se puso en contacto por vía telefónica con los responsables y el profesorado de educación física del centro, para explicarles los objetivos y procedimientos del proyecto y obtener los permisos pertinentes por parte de los padres para la recogida de las medidas antropométricas.

Posteriormente se concretó con los centros el calendario y las fechas en las que se procedió a la toma de datos, donde la mientras la mitad del alumnado rellenaba un cuestionario, a la otra mitad se le tomaba las medidas antropométricas. La realización de la toma de datos no duraba más de 45 minutos.

\section{Análisis estadístico de los datos}

En primer lugar, se realizaron análisis descriptivos por cada variable seleccionada en función del sexo. Y finalmente, se realizaron análisis correlaciónales mediante la $r$ de Pearson, para comprobar la asociación entre las dimensiones de IMC y la Competencia Percibida.

\section{Resultados}

Como se puede observar en la tabla 1 , los valores medios para el índice de masa corporal los valores medios son parecidos tanto en el grupo de los chicos como el de las chicas, y por lo tanto la diferencia entre los dos grupos no es significativa.

En cuanto a la percepción de competencia motriz, se encuentran diferencias significativas (ñ£.000) entre el grupo de las chicas y los

Tabla 1.- Valores medios delíndice de masa corporal en función del sexo.

\begin{tabular}{ccccc}
\hline & \multicolumn{2}{c}{$\boldsymbol{M ( D T )}$} & $\boldsymbol{t}$ & Sig. \\
& $\begin{array}{c}\text { Chicos } \\
\text { In= 173) }\end{array}$ & $\begin{array}{c}\text { Chicas } \\
(\mathrm{n}=188)\end{array}$ & $\boldsymbol{t}$ & \\
\cline { 2 - 6 } & $20.86(3.27)$ & $20.80(3.50)$ & .183 & .855 \\
\hline
\end{tabular}

Tabla 2.- Valores medios de competencia percibida en función del sexo.

\begin{tabular}{|c|c|c|c|c|}
\hline & \multicolumn{2}{|c|}{$M(D T)$} & \multirow[b]{2}{*}{$t$} & \multirow[b]{2}{*}{ Sig. } \\
\hline & $\begin{array}{c}\text { Chicos } \\
(\mathrm{n}=166)\end{array}$ & $\begin{array}{c}\text { Chicas } \\
(\mathrm{n}=185)\end{array}$ & & \\
\hline CP & $14.34(3.77)$ & $11.80(4.22)$ & 5.904 & .000 \\
\hline
\end{tabular}

Tabla 3.- Correlaciones entre las variables Índice de masa corporal y Percepción de competencia motriz

\begin{tabular}{ccc}
\multicolumn{3}{c}{ competencia motriz } \\
\hline ICM & ICM & PCM \\
PCM & $.162^{* *}$ & $.162^{* *}$ \\
\hline
\end{tabular}

chicos, siendo estos últimos los que tienen valores medios más altos. (Tabla2).

La correlación realizada demuestra que la relación entre el índice de masa corporal y la competencia percibida es significativa y negativa, es decir, a mayor índice de masa corporal peor percepción de la competencia motriz de cada uno (Tabla 3).

\section{Discusión}

Atendiendo a los resultado anteriormente expuestos, se observa que la relación que se da entre el IMC y la Percepción de competencia motriz es significativa y negativa, por lo que se supone que a mayor IMC peor percepción de la competencia motriz y a menor IMC mejor percepción de competencia motriz del alumnado muestreado. Los resultados obtenidos coinciden con los resultados derivados de diferentes estudios (Jones, Okely, Caputi \& Cliff, 2010; Southall, Steele \& Okely, 2004), en los que se afirma la estrecha relación que se da entre el IMC y la percepción de competencia percibida, tanto en chicos como en chicas, a pesar de ser un factor subjetivo.

El estudio llevado a cabo por el Cantell, Crawford y Doyle-Baker en 2008 ratifica esta afirmación. En este estudio observaron que los sujetos con valores más altos de IMC obtuvieron un nivel de percepción de competencia más bajo. Por consiguiente, podríamos determinar que la percepción de competencia puede ser un importante factor que contribuya al mantenimiento de la obesidad y el sobrepeso juvenil tal y como confirman Cantel, Crawford y Doyle-Baker.

Resumiendo y considerando los datos expuestos anteriormente y las diferencias entre los sexos (chicas y chicos), encontramos que respecto al índice de masa corporal, no hay diferencias entre los grupos, puesto que los dos obtienen valores parecidos. En cuanto a la percepción de competencia motriz, tal y como concluyen Raudsepp y Liblik (2002) en su investigación, los datos han mostrado que el grupo de los chicos se percibe más competente que el grupo de las chicas. Esto puede deberse a varios factores, entre otros la fuente de información en la que se basan para realizar su propia valoración (Horn \& Weiss, 1991; Weiss \& Amorose, 2005; Weiss, Horn \& Ebbeck, 1997).

Por otro lado, la comparación con el sexo opuesto, en este caso la comparación del nivel de competencia de las chicas con respecto al nivel de competencia de los chicos, puede estar basada en la concepción del estereotipo del hombre como modelo corporal enérgico, que posee mayores dotes y características para el trabajo físico (Antúnez, 2001). Por lo tanto, no es de extrañar, que se observen menores niveles de percepción de competencia en el grupo de las chicas.

\section{Conclusiones}

De los datos recabados, respecto a la competencia motriz percibida, podemos determinar que el $55.7 \%$ de los chicos de la muestra se perciben con un nivel medio de competencia y el 56\% de las chicas se perciben con un bajo nivel de competencia. En cuanto a las diferencias entre sexos, los chicos se perciben más competentes motrizmente que las chicas.

En relación al IMC, a modo de conclusión, se determina que en el caso de los chicos con sobrepeso y obesidad, el $47.8 \%$ se percibe con un medio nivel de competencia y un $32.1 \%$ con un bajo nivel de competencia. En cuanto a las chicas, el 67.8\% con sobrepeso y obesidad se perciben con baja competencia.

\section{Referencias}

Antúnez, M. (2001). Reflexiones acerca de lo que la mujer representa para el deporte y el verdadero significado del deporte para la mujer. Revista digital, 47, Buenos Aires.

Arruza, J.A., Arribas, S., Otaegi, O., González, O., Irazusta, S., \& Ruiz, L. M. (2011). Percepción de competencia, estado de ánimo y tolerancia al estrés en jóvenesdeportistas de alto rendimiento. Anales de psicología, 27, 2, 536-543. 
Biografía Adolphe Quételet (s.f.). Disponible en http:// docentes.educacion.navarra.es/mpastorg/cd_alumno/modeloG/ 2bach_CSS/Datos/biografias/14.pdf

Bueno, M., Ruiz, L. M., Graupera, J. L., \& Sánchez, F. (2001). Análisis comparativo de diferentes procedimientos de detección de los problemas evolutivos de coordinación motriz en los escolares de 4 a 6 años. Madrid: CIDE, Ministerio de Educación y Ciencia. Disponible en http://www.doredin.mec.es/documentos/ 080100003.pdf

Cantell, M., Crawford, S. G., \& Doyle-Baker P. K. (2008). Physical fitness and health indices in children, adolescents and adults with high or low motor competence. Human Movement Science Coordination Disorder, 27(2), 344-362

Causgrove Dunn, J. L., \& Watkinson, E. J. (1994). A study of the Relationship Between Physical Awkwardness and Children's Perceptions of Physical Competence. Adapted Physical Activity Quarterly, 11, 275-283.

Eaton, D. K., Kann, L., Kinchen, S., Ross, J., Hawkins, J., Harris, W. A. et al. (2006). Youth risk behavior surveillance—United States, 2005. Morbidity and Mortality Weekly Report, 55, 1-108.

Gómez, M. (2004). Problemas evolutivos de coordinación motriz y percepción de competencia en el alumnado de primer curso de educación secundaria obligatoria en la clase de educación física. Tesis doctoral. Madrid: Universidad Complutense de Madrid.

Henderson, S., \& Sugden, D. (1992): Movement assessment battery for children. Londres: The Psychological Corporation.

Horn, T. S., \& Weiss, M. R. (1991). A developmental analysis of children's self ability judgements in the physical domain. Pediatric Exercise Science, 3(4), 310--326.

Jones, R. A., Okely, A. D., Caputi, P., \& Cliff, D. P. (2010). Perceived and actual competence among overweight and non-overweight children. Sport Medicine Australia, 13(6), 589-596.

Lakka, T. A., \& Bouchard, C. (2005). Physical activity, obesity and cardiovascular diseases. En A. von Eckardestein (Ed.),
Spain. International Journal of Applied Sport Sciences, 15(1), $22-$ 35.

Ruiz, L. M., Graupera, J. L., Gutiérrez, M., \& Nishida, T. (2004). El testAMPETdemotivación de logro para el aprendizaje en educación física: Desarrollo y análisis factorial de la versión española. Revista De Educación, 335, 195-211.

Ruiz, L. M., Mata, E., \& Moreno, J. A. (2008). Problemas evolutivos de coordinación motriz y autoconcepto físico en escolares de educación primaria. Estudios de Piscología, 29(2), 163-172.

Santos, S. (2005). La educación física escolar ante el problema de la obesidad y el sobrepeso. Revista Internacional de Medicina y Ciencias de la Actividad física y el Deporte, 5(19), pp.179-199.

Solbes, I. (2010). El sobrepeso en la niñez y sus consecuencias sociales: un estudio evolutivo sobre las actitudes explícitas hacia la complexión y su relación con la imagen corporal. Tesis doctoral. Madrid: Universidad Complutense de Madrid.

Southall, J.E., Steele, J. R., \& Okely, A. D. (2004). Actual and perceived physical competence in overweight and nonoverweight children. Pediatric Exercise Science, 16(1), 15.

Weiss, M. R., \& Amorose, A. J. (2005). Children's Self-Perceptions in the Physical Domain: Between- and Within- Age Variability in Level, Accuracy, and Sources of Perceived Competence. Journal of Sporty Exercise Psychology, 27, 226-244.

Weiss, M. R., Horn, T. S., \& Ebbeck, V. (1997). Children’s SelfPerceptions and Sources of Physical Competence Information: A Cluster Analysis. Journal of Sport \& Exercise Psychology, 19, 5270.

\section{Nota del Autor;}

Nuestro más sincero agradecimiento a las siguientes entidades y respectivos profesores de la asignatura de Educación Física por la colaboración prestada, sin ellos no habría sido posible.

- Programa Predoctoral, de Formación de Personal Investigador no doctor del Departamento de Educación, Universidades e Investigación.
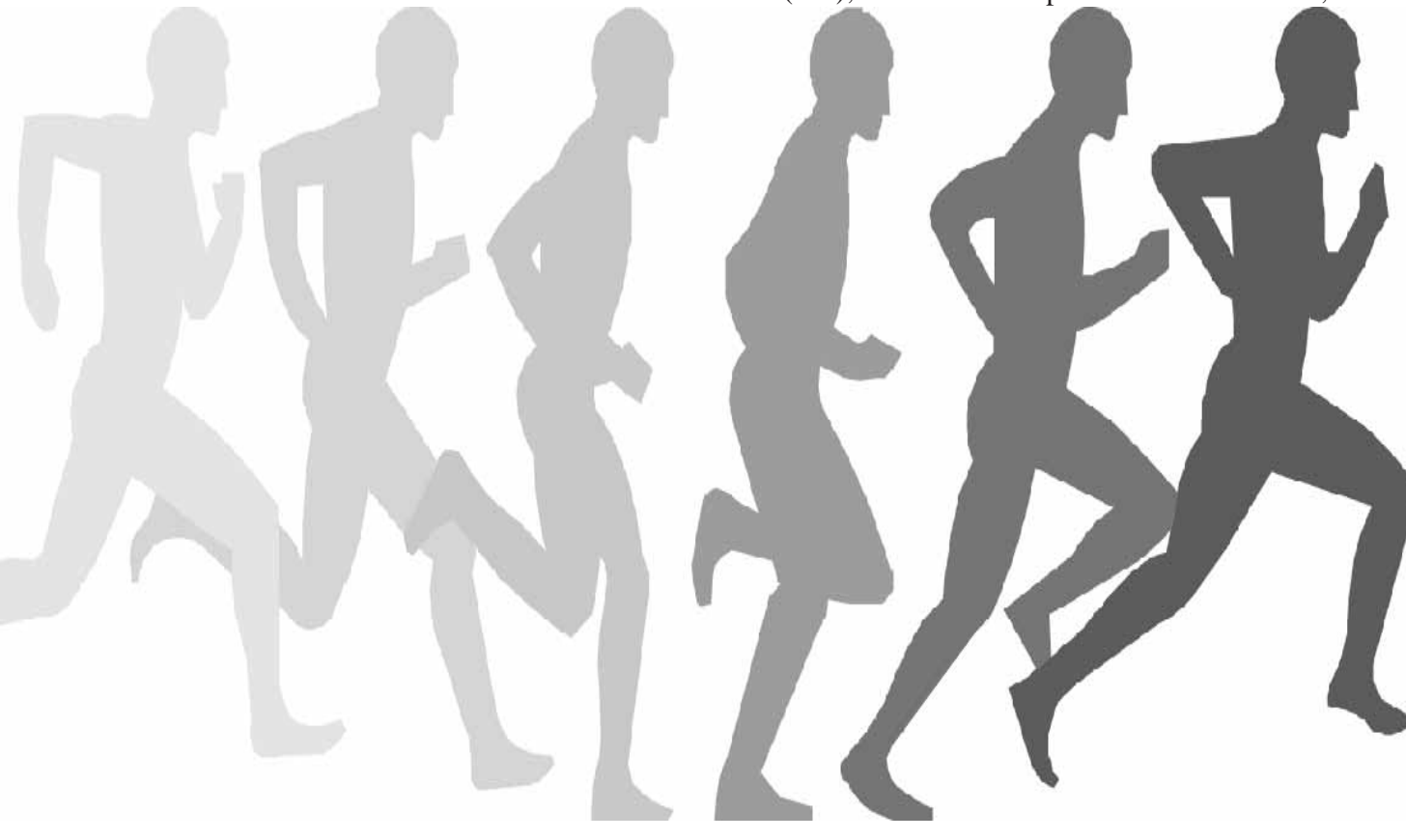

Sebastián), Arizmendi txea (Irún), Colegio El stola (Irún). los Pirineos(referencia ormación de Personal 